\title{
Long form collapsin response mediator protein-1 promotes the migration and invasion of osteosarcoma cells
}

\author{
HUIGE HOU $^{1 *}$, LIN CHEN $^{2,3^{*}}$, ZHENGANG ZHA $^{1}$, SHAOHUI CAI $^{2}$, MINGHUI TAN $^{4}$, \\ GUOQING GUO ${ }^{4}$, NING LIU ${ }^{1}$, GUORONG SHE ${ }^{1}$ and SONGWEI XUN ${ }^{1}$ \\ ${ }^{1}$ Department of Orthopedics, The First Affiliated Hospital; ${ }^{2}$ College of Pharmacy; ${ }^{3}$ Department of Pharmacy, \\ The First Affiliated Hospital; ${ }^{4}$ School of Medicine, Jinan University, Guangzhou, Guangdong 510632, P.R. China
}

Received January 7, 2015; Accepted January 19, 2016

DOI: $10.3892 / 01.2016 .4562$

\begin{abstract}
It has been reported that long form collapsin response mediator protein-1 (LCRMP-1) promotes the metastasis of non-small cell lung cancer. Osteosarcoma (OS) is a human cancer with a high potential for metastasis. The present study aimed to investigate the role of LCRMP-1 in OS metastasis. The expression of LCRMP-1 in OS specimens and cell lines was evaluated using reverse transcription-quantitative polymerase chain reaction (RT-qPCR) and western blot analysis. Furthermore, the migration and invasion of OS cells with LCRMP-1-knockdown was investigated to examine the role of LCRMP-1 in OS metastasis. In addition, the expression of $\mathrm{N}$-cadherin and matrix metalloproteinases (MMPs), which are involved in cell migration, was evaluated using RT-qPCR. Increased expression of LCRMP-1 was observed in the OS tissues and cell lines, accompanied by the enhanced migration and invasion of the OS cells. LCRMP-1-knockdown resulted in a significant decrease in the expression of $\mathrm{N}$-cadherin and MMPs, as well as inhibition of the migration and invasion of the OS cells. Overexpression of LCRMP-1 promoted OS metastasis. Therefore, LCRMP-1 may be a promising target for the effective treatment of OS.
\end{abstract}

\section{Introduction}

Osteosarcoma (OS) is the most common primary bone malignancy, occurring predominantly in the long bones of children and young adults. Long-term survival rates have improved

Correspondence to: Dr Zhengang Zha, Department of Orthopedics, The First Affiliated Hospital, Jinan University, 613 Huangpu Avenue West, Guangzhou, Guangdong 510632, P.R. China

E-mail: zhazhengang0107@163.com

Dr Shaohui Cai, College of Pharmacy, Jinan University, 601 Huangpu Avenue West, Guangzhou, Guangdong 510632, P.R. China

E-mail: 526122873@qq.com

*Contributed equally

Key words: long form collapsin response mediator protein-1, osteosarcoma, migration, invasion from $<20 \%$ to $65-70 \%$ following the advent of multiagent chemotherapy regimens (1). It is estimated that the annual incidence of osteosarcoma is 4-5 cases per 1,000,000 individuals worldwide (2). Due to its high propensity for distant metastasis (typically to the lungs), OS is one of the leading causes of cancer-associated mortality in adolescents (3). Metastasis, a major cause of cancer treatment failure and mortality, is a complex process in which various events are involved, including cell migration, angiogenesis, adhesion and cell proliferation (4). While the molecular mechanisms underlying cancer metastasis remain to be fully elucidated, it has been reported that a number of inducers and suppressors have significant roles.

Collapsin response mediator protein-1 (CRMP-1), a member of the CRMP family, is involved in cortical neuronal migration via reelin signaling (5). CRMP-1 has been observed to be a cancer invasion suppressor (6). In addition, a negative association between CRMP-1 expression and cancer cell invasiveness has been observed in non-small cell lung cancer (NSCLC) patients (7). Pan et al identified a novel isoform of CRMP family proteins in 2008, which was termed long form CRMP-1 (LCRMP-1) (8). In contrast to CRMP-1, which inhibits the migration and invasion of NSCLC, LCRMP-1 promotes filopodia formation, cancer cell migration and invasion, resulting in poor clinical outcomes in NSCLC patients (9). Therefore, LCRMP-1 is an invasion enhancer in NSCLC.

As OS has a high propensity for metastasis, and LCRMP-1 is able to promote the metastasis of NSCLC, it may be useful to identify whether LCRMP-1 is involved in the metastasis of OS. In the present study, the expression of LCRMP-1 was investigated and compared in OS patients and cell lines, as well as in a normal osteoblast cell line. In addition, migration and invasion assays were performed to investigate the migration and invasion of OS cells.

\section{Materials and methods}

Ethical approval. All human experiments were approved by the First Affiliated Hospital of Jinan University (Guangzhou, China). All experiments using human specimens were performed in accordance with the ethical standards set out in the 1964 Declaration of Helsinki and its later amendments. Informed consent was received from all participants prior to commencement of the study. 
Specimens. Formalin-fixed, paraffin-embedded tissue samples were obtained from 20 OS patients that received amputation at the Department of Orthopedics, First Affiliated Hospital of Jinan University (Guangzhou, China), between 1998 and 2013. Normal bone tissue from the same patient was used for control. Twenty patients with OS of the bone were selected from the files of the Department of Clinical Pathology at the Vienna General Hospital (Vienna, Austria). All cases were reviewed to confirm the diagnoses and to select a paraffin wax block for immunohistochemical studies.

Cell culture and generation of small interfering (si)RNA stable cell line. The human osteoblast hFOB 1.19 and OS cell lines SAOS2, MG63 and U2OS were supplied by the Cell Bank of the Shanghai Institute of Cell Biology, Chinese Academy of Sciences (Shanghai, China). The cells were maintained in Dulbecco's minimal essential medium (DMEM; Gibco; Thermo Fisher Scientific, Inc., Waltham, MA, USA) supplemented with $10 \%$ fetal bovine serum (FBS), penicillin $(100 \mathrm{U} / \mathrm{ml})$, and streptomycin $(100 \mu \mathrm{g} / \mathrm{ml})$ at $37^{\circ} \mathrm{C}$ in a humidified atmosphere with $5 \% \mathrm{CO}_{2}$.

A lentiviral (LV)-008 vector (Forevergen Biosciences, Guangzhou, China) with a U6 promoter was used to generate the recombinant lentivirus plasmid encoding small hairpin (sh)-LCRMP-1. The si-LCRMP-1 sequence was as follows: 5'-CAGCGAGGACACGGCCAGCGA-3'. HEK 293T cells (Forevergen Biosciences) were cotransfected with LV-008 and packaging vectors (Forevergen Biosciences), and the culture supernatant was harvested following 48 and $72 \mathrm{~h}$ of transfection. The OS MG63 cells were infected by sh-LCRMP-1 (shp300) and sh-scramble [negative control, (NC)] in the presence of 5-10 $\mu \mathrm{g} / \mathrm{ml}$ Polybrene (Santa Cruz Biotechnology, Inc., Dallas, TX, USA), respectively. After $48 \mathrm{~h}$, the cells were grown in DMEM supplemented with $10 \% \mathrm{FBS}, 100 \mathrm{U} / \mathrm{ml}$ penicillin and $100 \mu \mathrm{g} / \mathrm{ml}$ streptomycin with $2 \mu \mathrm{g} / \mathrm{ml}$ puromycin for 12 days, to generate OS MG63 sh-LCRMP-1 stable cell lines.

Migration assay. The cell migration assay was performed using Transwell cell culture chambers (EMD Millipore, Billerica, MA, USA). The OS MG63 cells were cultured in serum-free DMEM for $24 \mathrm{~h}$. The cells were subsequently trypsinized and resuspended in serum-free DMEM. The cells $\left(5 \times 10^{4}\right.$ per well) were seeded into the upper chamber of the Transwell insert and incubated with $0.5 \%$ dimethyl sulfoxide $(10 \mu \mathrm{M}$; Sigma-Aldrich, St. Louis, MO, USA) or deguelin (15 $\mu \mathrm{M}$; Sigma-Aldrich), and $90 \%$ DMEM medium containing $10 \%$ FBS was added to the lower chamber and incubated for $48 \mathrm{~h}$. Following incubation, the remaining cells in the upper chamber were removed. The migrated cells on the lower surface of the filter were fixed with $4 \%$ formaldehyde and stained with $2 \%$ crystal violet in $2 \%$ ethanol. The cells were subsequently counted and images were captured under a microscope (magnification, x200; Olympus BH-2; Olympus, Tokyo, Japan).

Invasion assay. The cell invasion assay was performed with similar methodology to the aforementioned migration assay, but with certain modifications. The filter membrane used in the invasion assay was coated with Matrigel (BD Biosciences). The cells on the lower surface of the filter were counted and images were captured under a light microscope (magnification, x200; Olympus BH-2). The invasive cells were fixed with $4 \%$ formaldehyde and stained with $2 \%$ crystal violet in $2 \%$ ethanol. Cell numbers were counted and expressed as the mean number of cells (mean \pm standard deviation). Experiments were performed in triplicate.

Immunohistochemistry (IHC). The specific rabbit anti-human anti-LCRMP-1 polyclonal antibody (C2) for western blotting and IHC was produced by the authors using a synthetic peptide derived from the unique N-terminal region of LCRMP-1, as described previously (6). Formalin-fixed, paraffin-embedded sections (5- $\mu \mathrm{m}$ thick) were processed for IHC. Antigen retrieval was performed with microwave treatment in $10 \mathrm{mM}$ citrate buffer ( $\mathrm{pH}$ 6.0). The endogenous peroxidase activity was quenched by $3 \%$ hydrogen peroxide for $10 \mathrm{~min}$. The sections were fixed using freshly prepared $4 \%$ paraformaldehyde, followed by permeabilization with $0.1 \%$ Triton X-100 in Tris-buffered saline and blocking in 5\% FBS. The antigen was incubated with LCRMP-1 (C2) antibody overnight at $4^{\circ} \mathrm{C}$. Detection of the immunoreactive staining was performed using the Super Sensitive ${ }^{\mathrm{TM}}$ Polymer HRP Detection system (BioGenex, Fremont, CA, USA) according to the manufacturer's protocols. The intensity of anti LCRMP-1 staining was semiquantitatively analyzed. Briefly, the number of positively stained cells and total number of cells in a given area were evaluated by two pathologists in a blinded manner. If the amount of positively stained cells was $\leq 5 \%$ the tissue was considered to be negative, $6-25 \%$ was weak expression (+); and $26-50 \%$ was moderate expression (++). A total of at least 400 cells from five areas of individual tissue samples were evaluated. The non-tumor bone was stained with anti-LCRMP-1 antibody.

Western blot analysis. Samples were lysed in sample buffer [(50 mM Tris-HCl, pH 6.8; 10\% glycerol, 2\% sodium dodecyl sulfate (SDS), $0.000125 \%$ bromophenol blue and 5\% $\beta$-mercaptoethanol] and subjected to SDS-polyacrylamide gel electrophoresis (10-40 $\mu \mathrm{g}$ protein of protein/lane), followed by incubation with rabbit anti-human anti-LCRMP-1 polyclonal antibody (C2), N-Cadherin (dilution, 1:1,000; catalog no., ab18203; Abcam, Cambridge, MA, USA), matrix metalloproteinase (MMP)-2 (dilution, 1:1,000; catalog no., ab37150; Abcam) and MMP-9 antibody (dilution, 1:1,000; catalog no., ab38898; Abcam). Glyceraldehyde-3-phosphate dehydrogenase (GAPDH; dilution, 1:2,500; ab9485, Abcam) was used as a loading control. Subsequent to being washed five times with phosphate-buffered saline containing $0.1 \%$ Tween 20 , the membrane was incubated with the goat anti rabbit $\operatorname{IgG}(\mathrm{H}+\mathrm{L})$ secondary antibodies (catalog no., G-21234; GE Healthcare Life Sciences, Uppsala, Sweden) for $1 \mathrm{~h}$, and bands were detected by enhanced chemiluminescence (Forevergen Biosciences).

Total RNA extraction and reverse transcription-quantitative polymerase chain reaction $(R T-q P C R)$. Total RNA was isolated from $5 \times 10^{6}$ cells using TRIzol ${ }^{\circledR}$ reagent (Invitrogen; Thermo Fisher Scientific, Inc., Waltham, MA, USA), according to the manufacturer's protocol. Initially, RNA was reverse transcribed using M-MLV Reverse Transcriptase (M1705; Promega Corporation, Madison, WI, USA) according to the manufacturer's protocol. qPCR was performed on a MiniOpticon $^{\text {TM }}$ Real-Time PCR system (Bio-Rad Laboratories, Inc., 

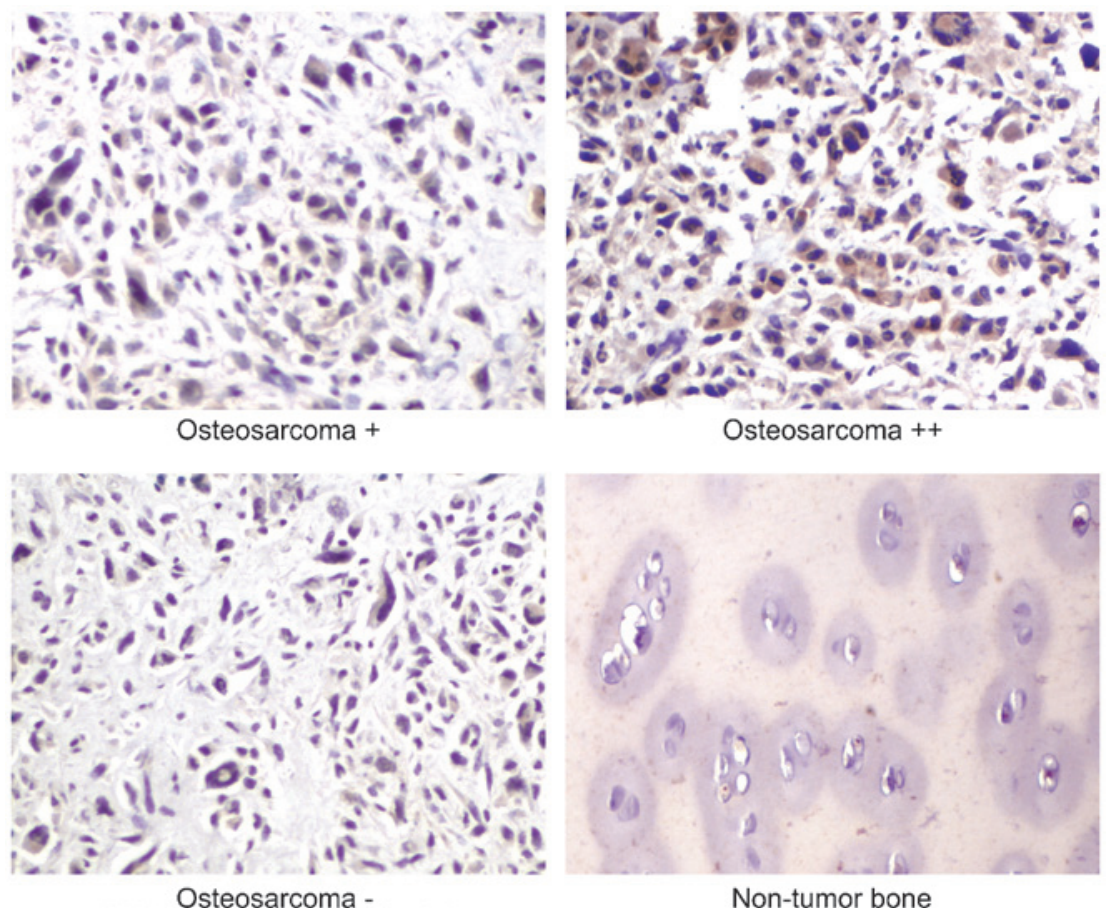

(without LCRMP-1 antibody)

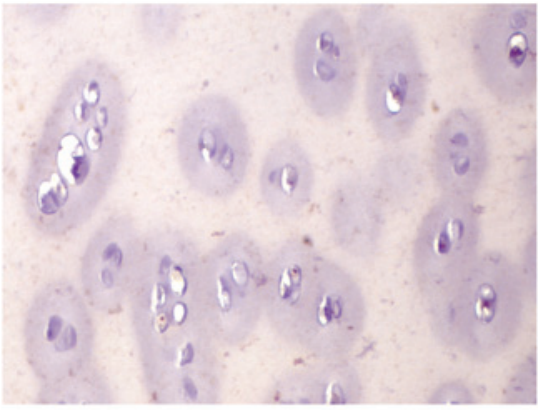

Non-tumor bone

expression of LCRMP-1 in Osteosarcoma

Figure 1. Increased LCRMP-1 expression in tumor tissues of osteosarcoma patients. Immunohistochemical staining of LCRMP-1 expression in normal and metastatic tissues at various levels. LCRMP-1 expression was defined as being immunoreactive to LCRMP-1 antibody and was increased in metastatic tissues compared to the osteogenic tissues. LCRMP-1, long form collapsin response mediator protein-1; -, negative ( $\leq 5 \%$ positively stained cells); +, weak (6-25\% positively stained cells); ++, moderate, $(26-50 \%$ positively stained cells).

Hercules, CA, USA) using $20 \mu 1$ reaction mixture, according to the manufacturer's protocol. The reagent used in qPCR was GoTaq $^{\circledR}$ qPCR Master Mix (A6002; Promega Corporation). GAPDH was the internal control (Gene ID, 2597). The oligonucleotide primers used were as follows: LCRMP-1 forward, 5'-GGAGGCAGCGAGGACAC-3' and reverse, 5'-CGTCAG CATAAAGGGATTGG-3'; and GAPDH forward, 5'-GAG TCAACGGATTTGGTCGT-3' and reverse, 5'GACAAGCTT CCCGTTCTCAG-3', provided by Forevergen Bioscience. The product signals were detected using SYBR Green. The PCR amplification program consisted of denaturation at $95^{\circ} \mathrm{C}$ in advance for $2 \mathrm{~min}$, followed by 40 cycles, each cycle consisting of denaturation at $95^{\circ} \mathrm{C}$ for $15 \mathrm{sec}$, annealing and extension at $60^{\circ} \mathrm{C}$ for $30 \mathrm{sec}$. The expression level of LCRMP-1 relative to that of GAPDH was defined as $2^{-\Delta \Delta \mathrm{Cq}}=2^{-[(\Delta \mathrm{Cq}) \text { LCRMP-1 }-(\Delta \mathrm{Cq}) \mathrm{GAPD}}$ ${ }^{\mathrm{H}]}$ (10). All experiments were repeated thrice.

Statistical analysis. All statistical analyses were performed using the SPSS 19.0 statistical software package (IBM SPSS, Armonk, NY, USA) Data are expressed as the mean \pm standard deviation. Statistical significance was determined by an analysis of variance. P-values of $<0.05$ were considered to indicated a statistically significant difference.

\section{Results}

Upregulation of LCRMP-1 in osteosarcoma specimens and cell lines. The expression of LCRMP-1 in OS and normal tissues was evaluated using IHC. The expression of LCRMP-1 in the OS specimens was significantly increased compared with that in normal bone tissues (Fig. 1). Western blot and RT-qPCR analyses were subsequently performed to evaluate the expression of LCRMP-1 at the protein and mRNA levels in the OS SAOS2, MG63 and U2OS cell lines. The results of the present study showed that LCRMP-1 mRNA and protein levels were significantly increased in the OS cell lines compared with the levels in osteoblast cells $(\mathrm{P}<0.05)$, and that the MG63 cells demonstrated the highest expression of LCRMP-1 (Fig. 2A and B). This suggested that LCRMP-1 was overexpressed in OS, and that it may have a significant role in OS development and progression.

To investigate the potential role of LCRMP-1 in the migration and invasion of OS cells, migration and invasion assays were performed in the OS SAOS2, MG63 and U2OS cell lines. As shown in Fig. 2, the migrated and invasive cells were stained, and the number of stained cells represented the migratory and invasive ability of the OS cells. Increased migration and invasion abilities were observed in the three OS cell lines. In addition, migration and invasion abilities were consistent with LCRMP-1 expression in each cell line. Among the three OS cell lines, the MG63 cells demonstrated the highest migration and invasion abilities $(\mathrm{P}<0.05$; Fig. 2). Therefore, the MG63 cell line was used in follow-up experiments to further investigate the role of LCRMP-1 in the migration and invasion of OS cells by knockdown of LCRMP-1.

Knockdown of LCRMP-1 suppresses OS cell migration and invasion. A stable LCRMP-1-knockdown cell line (sh-LCRMP-1) was established to further investigate the role of LCRMP-1 in OS metastasis. The role of LCRMP-1 
A

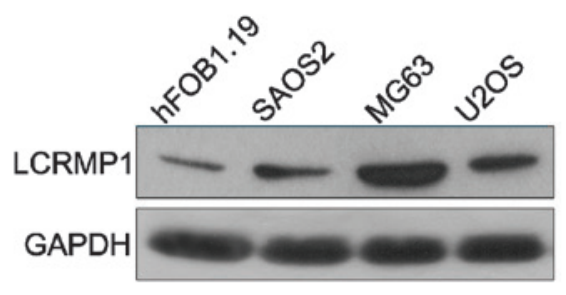

C

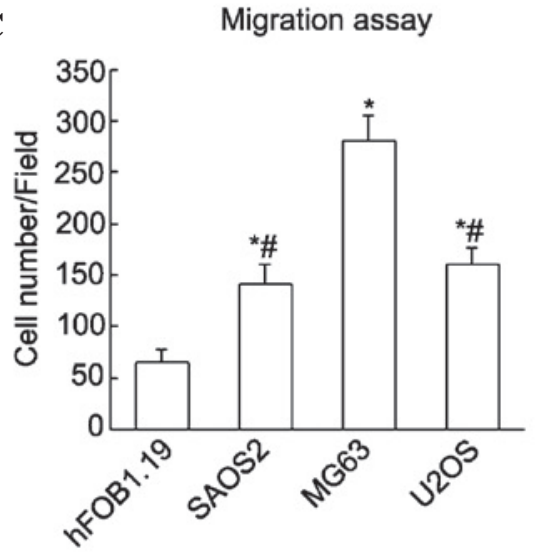

B

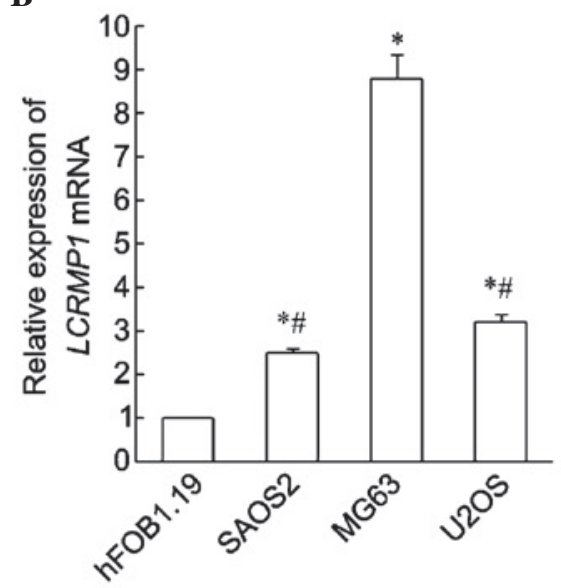

D

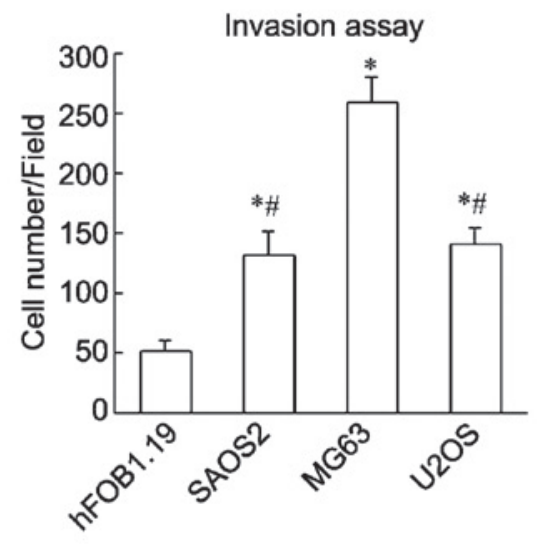

$\mathbf{E}$ hFOB1.19 SAOS2

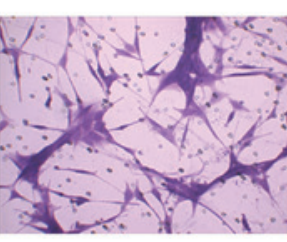
MG63 U2OS

Migration assay
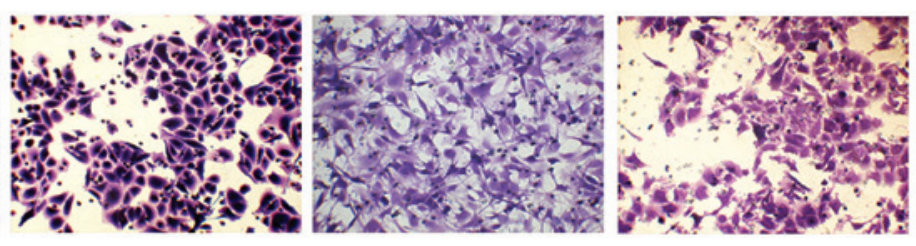

Invasion assay
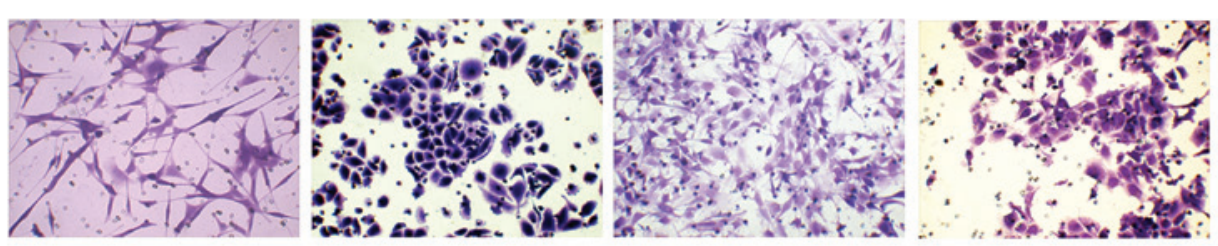

Figure 2. Invasion and migration ability in OS cell lines with varying expression levels of LCRMP-1. LCRMP-1 was upregulated in OS cells at the (A) protein and (B) mRNA levels. mRNA levels were confirmed by reverse transcription-quantitative polymerase chain reaction and protein levels were confirmed by western blotting. (C) Migration and (D) invasion ability in three OS cell lines, SAOS2, MG63 and U2OS, compared with the human osteoblast hFOB1.19 cell line. (E) Immunohistochemistry results are presented. Increased migration and invasion abilities were observed in the three OS cell lines. In addition, migration and invasion abilities were consistent with LCRMP-1 expression in each cell line. Among the three OS cell lines, the MG63 cells demonstrated the highest migration and invasion abilities. ${ }^{\mathrm{P}}<0.05$ vs. hFOB1.19. ${ }^{\#} \mathrm{P}<0.05$ vs. MG63. All experiments were performed in triplicate and the results are expressed as the mean \pm standard deviation, analyzed by analysis of variance. OS, osteosarcoma; LCRMP-1, long form collapsin response mediator protein-1; mRNA, messenger RNA; GAPDH, glyceraldehyde-3-phosphate dehydrogenase.

in tumor cell migration and invasion was examined in cells that constitutively expressed si-LCRMP-1 and in control cells. Western blot and RT-qPCR analyses showed that LCRMP-1 expression was significantly decreased in the sh-LCRMP-1 cells compared with that in the control and negative control cells (scramble) $(\mathrm{P}<0.05)$. In addition, reduced expression of migration and invasive factors, including N-Cadherin, MMP-2 and MMP-9, at the protein and mRNA levels was observed in the sh-LCRMP-1 cells ( $\mathrm{P}<0.05$; Fig. $3 \mathrm{~A}$ and $\mathrm{B})$. Migration and invasion assays were subsequently performed to evaluate the effects of LCRMP-1 downregulation on OS metastasis. As shown in Fig. 3C and D, LCRMP-1-knockdown significantly inhibited the migration and invasion of the MG63 cells compared with the control and scrambled cells $(\mathrm{P}<0.05)$. The 
A
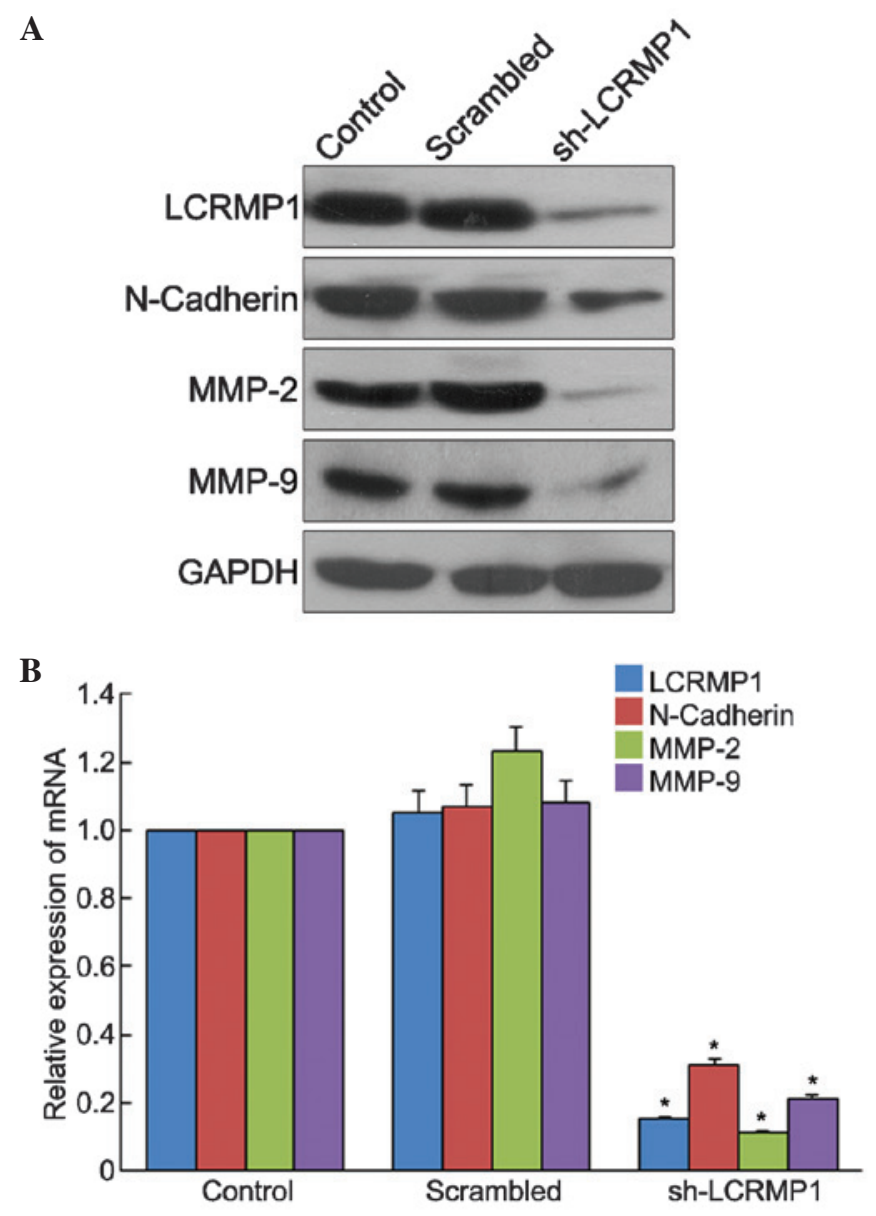

C
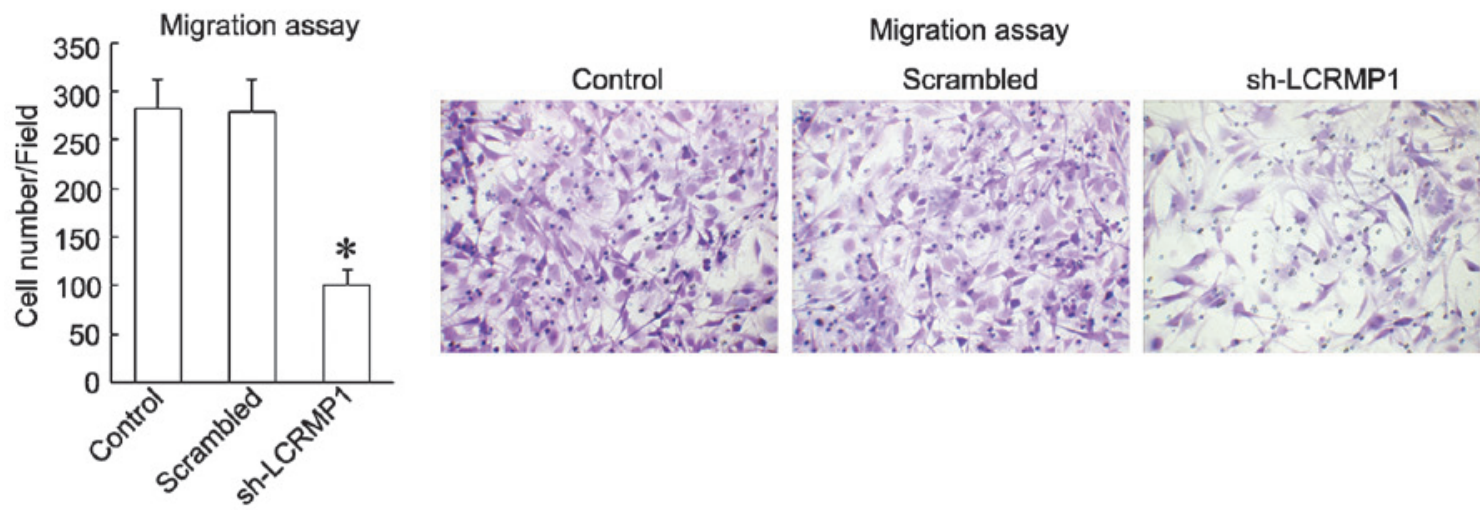

D
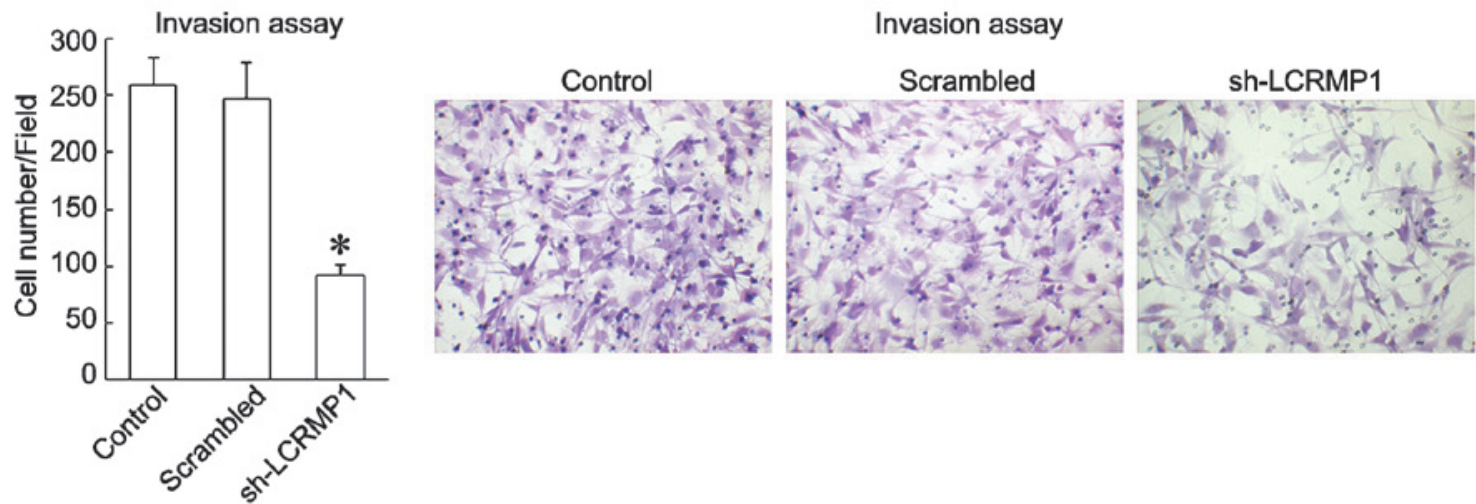

Figure 3. Reduced cell invasion and migration ability in LCRMP-1-knockdown cells. Downregulation of LCRMP-1, and invasive factors N-cadherin, MMP-2 and MMP-9 was detected in the sh-LCRMP-1 cells at the (A) protein and (B) mRNA levels. mRNA levels were confirmed by reverse transcription-quantitative polymerase chain reaction and protein levels were confirmed by western blotting. (C) Migration and (D) invasion ability in sh-LCRMP-1 cells. Decreased levels of migration and invasion ability were detected in the sh-LCRMP-1 cell lines. " $\mathrm{P}<0.05$ vs. control. All experiments were performed in triplicate and results are expressed as the mean \pm standard deviation, analyzed by analysis of variance. LCRMP-1, long form collapsin response mediator protein-1; MMP, matrix metalloproteinase; GAPDH, glyceraldehyde-3-phosphate dehydrogenase; sh, small hairpin; mRNA, messenger RNA. 
results of the present study suggested that LCRMP-1 promotes the migration and invasion of OS cells.

\section{Discussion}

Osteosarcoma is the most common primary bone malignancy in children and adolescents, accounting for $20 \%$ of all bone cancer cases. In total, $15-25 \%$ of OS cases metastasize (primarily to the pulmonary system), resulting in failure of anticancer treatments in OS patients. It has been reported that the 5-year disease-free survival of OS patients with metastasis is $10-20 \%$, while in patients without metastasis, the rate is $60-70 \%(11,12)$. However, the molecular mechanisms underlying OS metastasis largely remain to be elucidated, therefore, the identification of novel factors involved in OS metastasis has significant and potential applications in anticancer treatments for OS patients.

LCRMP-1 is a newly identified tumor enhancer in NSCLC patients. A previous study showed that LCRMP-1 overexpression promoted cancer cell invasion in vitro and in vivo, and that it was associated with poor overall and disease-free survival in NSCLC patients (8). It has also been shown that LCRMP-1 enhances filopodia formation and is phosphoregulated by glycogen synthase kinase $3 \beta$ in NSCLC cells $(9,13)$. The results of these previous studies led the present study to investigate the role of LCRMP1 in OS progression. In the present study, the results suggested that LCRMP-1 may promote the migration and invasion of OS cells.

The diagnosis of OS depends largely on biopsy and imaging examinations, and a failed early diagnosis of OS leads to delayed treatment (14-16). In addition, certain patients still suffer from metastases and other complications following aggressive treatments, including ablation and chemotherapy, suggesting that OS is highly resistant to chemotherapy and has a high potential to metastasize $(17,18)$. Therefore, novel approaches for diagnosis and novel drug targets are required. It has been reported that LCRMP-1 promotes the migration and invasion of NSCLC cells $(8,9)$. In addition, increased expression of $\mathrm{N}$-cadherin and MMPs following LCRMP-1 upregulation has confirmed that LCRMP-1 has a significant role in the migration, invasion and metastasis of tumor cells, as N-cadherin and MMPs are involved in epithelial-mesenchymal transition and remodelling of the extracellular matrix $(19,20)$. In the present study, overexpression of LCRMP-1 was detected in OS cell lines. Furthermore, knockdown of LCRMP-1 resulted in significant downregulation of N-cadherin and MMPs (MMP-2 and MMP-9), which was consistent with the significantly reduced number of OS cells with migratory and invasive properties. The results of the present study suggest that LCRMP-1 may be able to promote OS metastasis, possibly via activation of $\mathrm{N}$-cadherin and MMPs.

Previously, cancer cell lines derived from OS, including SAOS2, MG63 and U2OS, have been used as important models for studying the metastatic mechanisms of OS, through evaluating morphological behaviors and gene expression alterations (21). In the present study, the results indicated that LCRMP-1 may promote the migration and invasion of OS cells. However, the downstream and upstream events of LCRMP-1 overexpression in OS require investigation in future studies to further understand the underlying molecular mechanisms.

In summary, the present study showed that LCRMP-1 was overexpressed in OS specimens and cell lines. LCRMP-1 promoted OS metastasis, possibly through the activation of $\mathrm{N}$-cadherin and MMPs (MMP-2 and MMP-9). The results of the present study suggest that LCRMP-1 may be a potential target for the effective treatment of metastatic OS.

\section{References}

1. Isakoff MS, Bielack SS, Meltzer P and Gorlick R: Osteosarcoma: Current treatment and a collaborative pathway to success. J Clin Oncol 33: 3029-3035, 2015.

2. Mirabello L, Troisi RJ and Savage SA: Osteosarcoma incidence and survival rates from 1973 to 2004: Data from the Surveillance, Epidemiology, and End Results Program. Cancer 115: 1531-1543, 2009.

3. Ta HT, Dass CR, Choong PF and Dunstan DE: Osteosarcoma treatment: State of the art. Cancer Metastasis Rev 28: 247-263, 2009.

4. Steeg PS: Tumor metastasis: Mechanistic insights and clinical challenges. Nat Med 12: 895-904, 2006.

5. Yamashita N, Uchida Y, Ohshima T, Hirai S, Nakamura F, Taniguchi M, Mikoshiba K, Honnorat J, Kolattukudy P, Thomasset $\mathrm{N}$, et al: Collapsin response mediator protein 1 mediates reelin signaling in cortical neuronal migration. J Neurosci 26: 13357-13362, 2006.

6. Shih JY, Lee YC, Yang SC, Hong TM, Huang CY and Yang PC: Collapsin response mediator protein-1: A novel invasion-suppressor gene. Clin Exp Metastasis 20: 69-76, 2003.

7. Shih JY, Yang SC, Hong TM, Yuan A, Chen JJ, Yu CJ, Chang YL, Lee YC, Peck K, Wu CW and Yang PC: Collapsin response mediator protein-1 and the invasion and metastasis of cancer cells. J Natl Cancer Inst 93: 1392-1400, 2001.

8. Pan SH, Chao YC, Chen HY, Hung PF, Lin PY, Lin CW, Chang YL, Wu CT, Lee YC, Yang SC, et al: Long form collapsin response mediator protein-1 (LCRMP-1) expression is associated with clinical outcome and lymph node metastasis in non-small cell lung cancer patients. Lung Cancer 67: 93-100, 2010.

9. Pan SH, Chao YC, Hung PF, Chen HY, Yang SC, Chang YL, Wu CT, Chang CC, Wang WL, Chan WK, et al: The ability of LCRMP-1 to promote cancer invasion by enhancing filopodia formation is antagonized by CRMP-1. J Clin Invest 121: 3189-3205, 2011.

10. Livak and Schmittgen: Analysis of relative gene expression data using real-time quantitative PCR and the $2-\Delta \Delta \mathrm{Ct}$ method. Methods 25: 402-408, 2001.

11. Marina N, Gebhardt M, Teot L and Gorlick R: Biology and therapeutic advances for pediatric osteosarcoma. Oncologist 9: 422-441, 2004.

12. Meyers PA and Gorlick R: Osteosarcoma. Pediatr Clin North Am 44: 973-989, 1997.

13. Wang WL, Hong TM, Chang YL, Wu CT, Pan SH and Yang PC: Phosphorylation of LCRMP-1 by GSK3 $\beta$ promotes filopoda formation, migration and invasion abilities in lung cancer cells. PLoS One 7: e31689, 2012.

14. Klein MJ and Siegal GP: Osteosarcoma: Anatomic and histologic variants. Am J Clin Pathol 125: 555-581, 2006.

15. Harada S, Wei S and Siegal GP: Molecular pathology of osteosarcoma. In: Bone Cancer. Primary Bone Cancers and Bone Metastases. Heymann D (ed). 2nd edition. Academic Press, Cambridge, MA, pp213-222, 2014.

16. Tirino V, Paino F, Papaccio F, La Noce M, Papaccio G and Desiderio V: Stemness markers of osteosarcoma. In: Bone Cancer. Primary Bone Cancers and Bone Metastases. Heymann D (ed). 2nd edition. Academic Press, Cambridge, MA, pp205-211, 2014.

17. Meyers PA, Schwartz CL, Krailo M, Kleinerman ES, Betcher D, Bernstein ML, Conrad E, Ferguson W, Gebhardt M, Goorin AM, et al: Osteosarcoma: A randomized, prospective trial of the addition of ifosfamide and/or muramyl tripeptide to cisplatin, doxorubicin, and high-dose methotrexate. J Clin Oncol 23: 2004-2011, 2005.

18. Janeway KA and Grier HE: Sequelae of osteosarcoma medical therapy: A review of rare acute toxicities and late effects. Lancet Oncol 11: 670-678, 2010.

19. Bryan RT and Tselepis C: Cadherin switching and bladder cancer. J Urol 184: 423-431, 2010.

20. Patel S, Sumitra G, Koner BC and Saxena A: Role of serum matrix metalloproteinase-2 and -9 to predict breast cancer progression. Clin Biochem 44: 869-872, 2011.

21. Walkley CR: Modeling osteosarcoma: In vitro and in vivo approaches. In: Bone Cancer. Primary Bone Cancers and Bone Metastases. Heymann D (ed). 2nd edition. Academic Press, Cambridge, MA, pp195-204, 2014. 